\title{
“There's just no flexibility": How space and time impact mental health consumer research
}

Brett Scholz ${ }^{\mathrm{a}}$, Sarah Gordon ${ }^{\mathrm{b}}$, Julia Bocking ${ }^{\mathrm{a}}$, Jackie Liggins ${ }^{\mathrm{c}}$, Peter Ellis ${ }^{\mathrm{b}}$, Cath Roper ${ }^{\mathrm{d}}$, ,

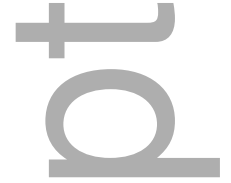

Chris Platania-Phung ${ }^{\mathrm{a}}, \&$ Brenda Happell $^{\mathrm{a}}$

${ }^{a}$ SYNERGY Nursing and Midwifery Research Centre, University of Canberra and ACT

Health, Canberra, Australia

(brett.scholz@canberra.edu.au; chris.platania-phung@canberra.edu.au;

julia.bocking@canberra.edu.au; brenda.happell@ canberra.edu.au)

${ }^{\mathrm{b}}$ Department of Psychological Medicine, University of Otago, Christchurch, New Zealand (sarah.e.gordon@otago.ac.nz; pete.ellis@ otago.ac.nz)

${ }^{c}$ Social and Community Health, The University of Auckland, Auckland, New Zealand (jackie.liggins@auckland.ac.nz)

${ }^{\mathrm{d}}$ Department of Nursing, The University of Melbourne, Melbourne, Australia (croper @ unimelb.edu.au)

\section{Corresponding Author}

\section{Dr Brett Scholz}

SYNERGY Nursing and Midwifery Research Centre

University of Canberra and ACT Health

The Canberra Hospital

Garran ACT 2605

Australia

email: brett.scholz@canberra.edu.au

phone: $+61(2) 62442197$

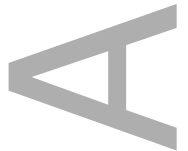

\section{Funding Sources}

This is the author manuscript accepted for publication and has undergone full peer review but has not been through the copyediting, typesetting, pagination and proofreading process, which may lead to differences between this version and the Version of Record. Please cite this article as doi: $10.1111 /$ INM.12589

This article is protected by copyright. All rights reserved 
This research did not receive any specific grand from funding agencies in the public, commercial, or not-for-profit sectors.

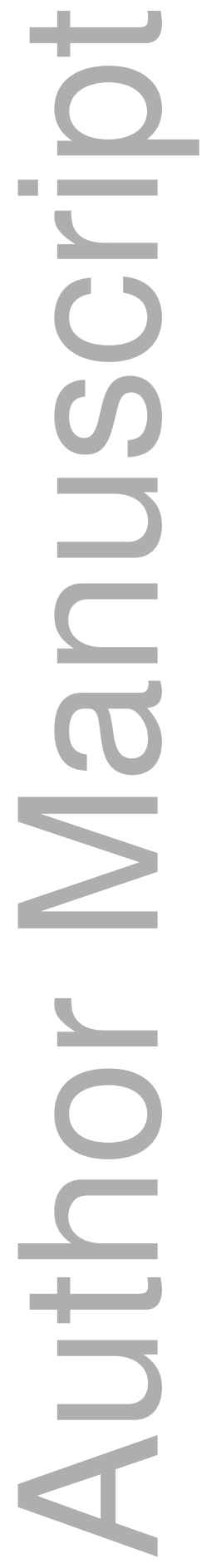




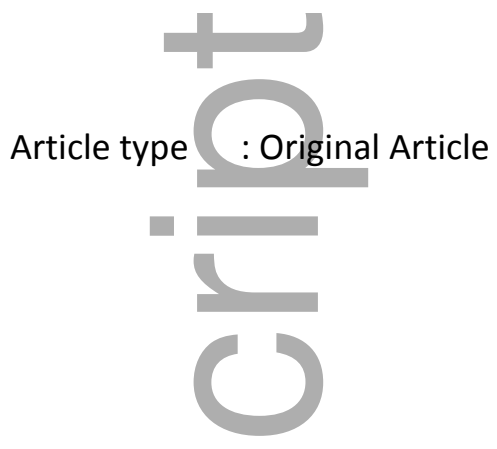

Non-consumer researchers collaborating with consumer researchers can benefit from greater relevance of research, and improved congruence between research processes and health policy. As with all research collaborations, such partnerships are both constrained and facilitated by research ecosystems. However, it seems that collaborations with consumer researchers are impacted in particular ways by the research ecosystem. Drawing on ecological systems theory, this study aims to improve understandings of how ecological structures impact collaborations between non-consumer and consumer researchers. Interviews were conducted with 11 non-consumer researchers from a range of mental health disciplines about their experiences collaborating with consumer researchers. One theme developed through analysis of the data set related to the research ecosystem. Data from this theme were extracted and discursively analysed using the principles of discursive psychology. Findings emphasise distinct factors that influence collaborations at each level of the ecosystem, encompassing both local research culture and broader research systems. Findings suggest that external pressures (such as deadlines for funding applications, or bureaucratic processes) from the broader ecosystemic levels need to be challenged at the local collaboration level. Non-consumer researchers might support collaborations through, for instance, working to create enhanced flexibility in research timelines, or making time for relationship building, thus fostering more meaningful collaborations.

Keywords: allyship, collaboration; consumers; consumer research; ecosystems; research 


\section{INTRODUCTION}

High quality mental health research is pivotal to improving the lives of people with mental ill health. As with other domains of health, however, it is increasingly seen as problematic that those without first-hand experience of mental illness determine the what and how of research. For instance, research led by people without lived experience of mental illness and/or mental health service usage has been described as less likely to be 'in touch' with the health needs, values and understandings of those with such experiences (Minogue \& Girdlestone, 2010). Furthermore, the talents and other qualities of people experiencing mental illness that contribute to strengthening mental health research may be overlooked because of pervasive stigma and discrimination (Scholz, Bocking, \& Happell, 2017a, 2017b; Scholz, Stewart, Bocking, \& Happell, 2017). A promising development has been the rise of consumer research (a term used throughout this paper to refer to research led by or co-created with mental health service consumers) (Michalak et al., 2016), however research points to significant barriers particularly related to resources, such as the time and funding necessary to facilitate consumer research (Staley, 2012). This paper will explore these barriers through a focus on how they are structured by relationships between ecological systems.

\section{BACKGROUND}

There are many examples of research where consumers are consulted or included in specific roles (such as conducting interviews), but research in which consumers collaboratively design and undertake research with other researchers as equal partners or research leaders is less common (see, for example, INVOLVE, 2012). In this paper we use the term 'other' researchers to refer to research members of traditional mental health fields such as psychiatry, psychology, social work and mental health nursing. As shorthand, the collaborative research between consumers and other mental health researchers, will be called 'co-created research'.

Literature based on completed consumer research projects and studies point to the importance of consumer input in research and especially the value of co-created mental health research (Ochocka, Janzen, \& Nelson, 2002; Roper, Grey, \& Cadogan, 2018;

Schneider, 2012). Consumer researchers often contribute insightful interpretations of research data about mental ill health that could only come from experiential expertise, and these can provide strengthened understandings of key phenomena or issues at play (Michalak et al., 
2016; Mjosund et al., 2017). The broader 'agendas' of mental health research, in particular the experience of mental illness and service evaluation, are often re-directed by consumers in ways that cover a broader spectrum of topics and issues than those arising from traditional disciplines such as psychiatry (Graham, Rose, Murray, Ashworth, \& Tylee, 2014; Michalak et al., 2016). Further, consumer perspectives have been described as encouraging more critical thinking about research than traditional disciplines (Veseth, Binder, Borg, \& Davidson, 2017). In addition, in several projects it was found that consumer participants were considerably more comfortable participating in research when a consumer researcher was present, for example, during interviews (Case et al., 2014). Similar benefits have arisen from consumer research organised around other identities of health or illness (Lowes \& Hulatt, 2005).

While consumer research is increasingly recognised, it remains relatively 'on the margins' from the point of view of mental health research overall. Historically, people labelled as having mental illness have been in the research space primarily as participants to be researched (Wallcraft, Schrank, \& Amering, 2009). Only in the last four decades have those with mental illness occupied research space as people who conduct research themselves or are involved in research co-creation (Landry, 2017; Rose, 2017). The minimal progress in centring consumer research in mental health has been attributed to multiple barriers revealed primarily through small-sample qualitative interview studies. As well as resources such as time and funding, mental illness stigma, unclear policy, and tokenism have also been highlighted in the peer-reviewed literature (Gee, McGarty, \& Banfield, 2016).

Studies have tended to explore barriers within specific projects (e.g., Bryant et al., 2012) or disciplines (e.g., Fothergill, Mitchell, Lipp, \& Nortway, 2012). While studies of the perspectives of consumer researchers have been important in understanding the research environment (Patterson, Trite, \& Weaver, 2014), less is known about the perspectives of other mental health researchers. This seems particularly important to understand as other researchers can have a fundamental role in facilitating consumer involvement (Happell \& Scholz, 2018; Juntanamalaga, Roper, Happell, \& Scholz, 2019). Additionally, the role of the research environment as a whole, in constraining or facilitating consumer research, is yet to be explored.

\section{Theoretical Framework}


Although some research has begun to examine the ways in which partnerships between consumer researchers and other mental health researchers operate, there is limited understanding of how such partnerships can be facilitated. Anecdotal evidence suggests a range of barriers that prevent such partnerships from reaching their full potential. To study these partnerships, it is valuable to look not only at the partnership itself, but also consider the broader contexts in which the partnership is located. The current study adopts ecological systems theory (Darling, 2007) as a lens through which the results are interpreted.

An ecological systems theory approach suggests that although partnerships are defined by interactions between consumer researchers and other mental health researchers at an individual level, they can better be understood by studying the multiple contexts in which partnerships occur. These contexts include the microsystem (the layer closest to the partnership), the mesosystem (the layer connecting structures of the microsystem), the exosystem (the larger social system), the macrosystem (the outermost layer in the environment), and the chronosystem (encompassing the dimension of time as it relates to each layer of the ecosystem) (Darling, 2007).

Within the context of partnerships between consumer researchers and other mental health researchers, the microsystem includes space immediately surrounding the partnership, such as offices where partners meet, or immediate outputs of research activities. The mesosystem includes interrelations among these microsystem structures, such as motivations for a particular partnership, or how research outputs influence ongoing partnerships. At the exosystemic level, contexts that affect the partnerships include research communities such as groups within particular universities, or collaboration networks. The macrosystem in this context might include ideologies of consumer-leadership, or requirements of policy or funding bodies. Last, the chronosystem in the context of such partnerships may include changes in collaborations and practices as they relate to time.

\section{Aims and Objectives}

This research, a collaboration between consumer and other mental health researchers, set out to explore the experiences of other mental health researchers, working in collaboration with consumer researchers. Although not the original aim of this study, data has been reinterpreted through an ecological framework. The study explores how ecological structures influenced these collaborations. To achieve this aim, there are three specific objectives: 
1. To conduct interviews with other mental health researchers to collate a range of experiences of collaborations with consumer researchers;

2. To examine other mental health researchers' discourses of constraints and facilitators of collaboration with consumer researchers through an ecological systems lens; and

3. To analyse the influence of the ecological systems of research on other researchers' experiences of collaboration.

-

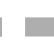

\section{METHODS}

\section{Design}

The current study used an exploratory, qualitative approach (Stebbins, 2001), given how little is understood about the area and the need to explore participants' understandings.

\section{Participants}

Snowball sampling was used to recruit other mental health researchers from Australia and New Zealand who had co-produced research with consumer researchers. Eleven participants agreed to be interviewed, representing a range of disciplinary backgrounds including social work, psychology, psychiatry, and mental health nursing. Participants represented a range of experience in mental health research (including, for example, senior professors and early career researchers), and positions of responsibility (including project leads, $\mathrm{PhD}$ supervisors, research consultants). Most participants discussed being involved in different kinds of collaborations with consumers at different times including as co-producers of research projects, in an advisory capacity, or in collaborating on single aspects of research studies.

\section{Ethics}

This study was approved by the [ethics committee hidden for review]. Participants were provided with information about the study, and all provided consent to have their interviews recorded and transcribed. Participants understood that participation was voluntary and that they were able to withdraw at any time.

\section{Data Collection}

Data collection was undertaken during 2017. Interviews included a range of questions about collaborating with consumers, but were largely open-ended to allow participants to drive the direction of the interview and discuss issues most salient to their experiences. Interviews were conducted by video or teleconferencing, or face-to-face. Interviews were 
approximately 60 minutes in length. Data were transcribed by an independent transcription company.

\section{Data Analysis}

The interview data were analysed for potential underlying themes, drawing on the guidelines provided by Braun and Clarke (2006). One theme developed during this process was about the ecological system in which research took place. All data coded to this particular theme was then analysed using the principles of discursive psychology (Potter, 2012). As such, analysis attended to the action-orientation of participants' accounts of research partnership (i.e. what particular discourses achieve, and who they privilege or marginalise), and the constructed and constructive nature of discourse (i.e. how particular individuals and actions are constructed by discourse, and how participants draw on and reproduce existing interpretive repertoires).

\section{RESULTS}

Our findings suggest that the strength of the collaboration between consumer and other mental health researchers is dependent on ecological structures. Such collaboration is complex, and consideration of relationships between layers of the ecosystem is important to better understand how meaningful and productive collaboration occurs. Following a theorydriven approach to analysis, the findings are presented in relation to each layer of the ecological systems from microsystem through to macrosystem.

\section{Microsystem}

At the layer closest to the relationship between consumer researcher and other mental health researchers, participants spoke about three particular sites of collaboration: building the relationship, impact of mental state, and nurturing skills.

\section{Building relationships}

At the microsystemic level, participants' accounts of working with consumer researchers focused on space that fostered relationship building, such as in Extract 1.

\section{Extract 1}


"I guess I'm quite lucky in that I work quite closely with somebody who has a dedicated position because of her experience and research expertise....I think positions like that are quite rare and so having somebody who is working in a dedicated research position, is not super common, so, I think that's one particular challenge."

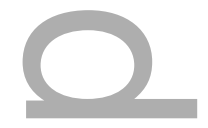

This participant notes that for most working relationships between consumer researchers and other mental health researchers, there is a "particular challenge" that there are few "dedicated research position[s]" for consumer researchers. Thus, other researchers often lack a space that fosters the ability to "work quite closely with" consumer researchers, and thus build relationships.

Indeed, being able to negotiate over joint research decisions, and to develop collective viewpoints was seen as a fundamental consideration for some participants. For example, another participant talked about how it "takes a lot of time" to work towards a "collaborative consensus" when bringing "together two groups who...have some history". Thus, at this level of the ecosystem, having the space and time to discuss research and form new relationships is vital.

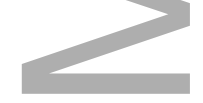

\section{Impact of mental state}

Participants were oriented to consumer researchers' mental health and capacity to complete research tasks on time as an important consideration within the microsystemic level of the relationship. The dominant narrative about consumer researchers has often been about consumers lacking capacity to collaborate. In most cases, participants in the current study challenged this narrative, stressing that 'unreliability' within projects was something 'to be dealt with' for both other researchers as well as consumer researchers (as in Extract 2), or that ensuring consumers be considered as part of the study design mitigated any such concerns (as in Extract 3).

\section{Extract 2}


“...certainly I've worked with people who aren't consumers who have been pretty shocking with deadlines and...so I don't think it's anything to do with that. I think it's more a matter of resources..."

\section{Extract 3}

"I can't say that's been a problem. Like I've been involved in a number of studies where we've have had service users involved and because they've built into the research design, if there's time involved in it then that's part of a study..."

Although such accounts demonstrate positive working relationships with consumers, it is of note that participants oriented to the mainstream view that collaboration with consumers can be challenging. Thus, within collaborative microsystems, this stigmatised perception of consumers could need addressing so that long-term, meaningful relationships can be fostered.

\section{Nurturing research skills and experience}

Participants mentioned that it was common for consumers to be entering research activities for the first time. The following extract is from a participant who saw nurturing research skills as part of their role in working with consumer researchers.

\section{Extract 4}

“..a novice needs things. They need extra resources and an environment and support, and probably more time. There are reasons why always working with people who are starting out is more challenging. But then I do quite like that, because I've done a lot of supervision and I really like the idea of growing capacity."

As this participant notes, a microsystem that facilitates "extra resources", "support", and "time" is one that can be successful in building consumer researchers' capacity. Similar to Extract 2, above, it is of note that these resources are not only needed by consumer 
researchers, but any novice researcher. Rather, as consumer researchers may have had fewer academic opportunities, the research partnership microsystem needs to accommodate this for successful, sustainable research outcomes.

\section{Mesosystem}

The mesosystemic level includes the structures and relationships between elements of the microsystem. As such, participants' accounts of collaboration mesosystems focused on how the relationships between consumer researchers and other mental health researchers were sustained by pacing and flexibility of projects, and structures that facilitated bringing together research collaborators.

\section{Pacing and flexibility}

Extract 3, above, considered how in the relationship microsystem, building consumer researchers into the study design mitigated potential timeline concerns. At the mesosystemic level, participants' accounts expanded on this, by exploring how projects could incorporate more flexibility. One participant, for example, noted that consumer researchers did not "necessarily want to do a seven o'clock start or an eight o'clock meeting" rather they would be "working to the hours that do work for them". Collaborators who challenge typically inflexible research environments, such as shifting to more feasible timelines, was one way that flexibility could be incorporated into research work. A more inclusive and effective pace for research projects is likely to foster enhanced participation from a diverse demographic of researchers, including but not limited to consumer researchers.

\section{Bringing together}

Given the considerable lack of consumer researchers working in dedicated research roles (see Extract 1), participants talked about how their research partnerships were creative in bringing collaborators together, such as in the following extract.

\section{Extract 5}

"...there is a reasonable pool of people who would be interested in going on that group. But...they might be quite spread around, I suppose, dotted around, rather than concentrated in a particular place. So, I guess, for that reason it might be better to have it as a virtual group." 
Similar views were common among participants. A "virtual group" that could meet via the internet, collaborate over email, and discuss projects remotely provided a microsystem that provided an optimal environment to meet research objectives. Particularly as consumer researchers often had fewer resources, and thus time and opportunities to travel to collaborate would be limited, virtual groups represented a possible way to bring teams together.

\section{Exosystem}

The exosystem comprises the broader contexts affecting research partnerships. Data from our interviews included the exosystemic structures of research culture and research funding.

\section{Research Culture}

At the mesosystemic level, participants noted the need for better pacing of projects and flexibility with time. In part, the inflexibility of research work is driven by an exosystemic research culture that demands too much commitment from researchers. The following extract comes from a participant questioning the assumption that researchers should be meeting demands at the cost of their wellbeing.

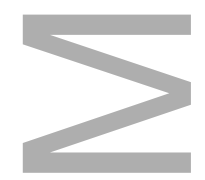

\section{Extract 6}

"Maybe we need to see that as a strength, that these people are actually saying 'I'm not going to die for this cause, I need to take time out," whereas we tend to die for the cause, and I don't know that, in the long run, that does us much good really, or the cause either for that matter."

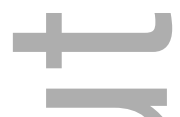

Overexertion is part of contemporary research culture, but as this participant suggests, relationships that allow collaborators to challenge such entrenched cultural norms may result in more attitudinal shift within researchers, as well as the research itself. Indeed, several participants noted that consumer researcher collaborators often had better ideas about how to negotiate these cultural norms than other mental health researchers. One said "actually I've learned from [consumer researchers]" about how to be more "competent at saying I feel I'm 
this close to my limit of what I can do", emphasising how they had learned from consumer researcher collaborators about how to manage some of the exosystemic pressure.

Another exosystemic factor that shapes research culture related to the external inflexibility of timelines determined by research funders, discussed in the following extract.

\section{Extract 7}

"There is an expectation that, well some things like tender deadlines, there's just no flexibility. Grant deadlines, various things. They're incredibly nonhuman. They're machine like, and you have to suck it up and do it."

The account in Extract 7 elaborates on some of the rigid structures at the exosystemic level than could lead to inflexibility at the research partnership level. Deadlines for grants, tenders, and ethics applications were criticised by several participants for being "nonhuman". Although this participant suggests that the inevitability of this rigidity: "you have to suck it up and do it", this may still be problematic for researchers needing more flexibility to best foster consumer participation in the research. Similarly, time is needed to build relationships at the microsystemic level (as in Extract 1). These structures can impinge on meaningful time for bringing together research teams for formal and social gatherings. The relative isolation is particularly problematic for consumer researchers who may not be in dedicated research positions, and thus do not have the time nor space to collaborate meaningfully on "machinelike" parts of the research process.

\section{Macrosystem}

At the broadest level of the ecosystem, participants oriented to the way that a number of ideological positions framed their relationships with consumer researchers. Consumer leadership or co-ereation within the mental health sector more broadly is still not well recognised. The following extract discusses several macrosystemic issues (including research not being valued, and professional silos) that impact research collaboration between consumers and other mental health researchers.

\section{Extract 8}


“...working for, within government agencies where you have so many steps to go through to have approvals or sign something off, that coupled with lack of time is somewhat of a disincentive to finish that bit off. Or sometimes it's because somebody - groups and units - has a concern about it. Like, one of the reasons why we [partnering government organisation names] didn't publish a number of things is that they thought it was too densely presented, didn't want to put their name behind it. And nobody had the time to write a summary or precis on it...then nobody has kind of got the time to go back to say to that writer or researcher it's not in the format that's fit for our purpose. And most of them [data] have got such nuggets in them, and because they're not available to other people studying in that sector, or working in that sector, it's an incredible waste...I think it's the complexity as well as time and those things go together."

With experience in government agencies, this participant had gained a strong sense of how the macrosystem impacted collaborations. One element at this level is the bureaucratic requirements both on a practical level ("so many steps to go through to have approvals") and in regards to preferred written report formats ("not in the format that's fit for our purpose"). Another macrosystemic structure is whether the work is valued or not ("nobody had the time to write a summary") and disagreements between collaborators on projects leading to projects ending without dissemination ("didn't publish [because some] didn't want to put their name behind it")

Extract 8 also refers to another macrosystemic structure: the barriers within the sector (good data "not available to other people...in that sector"). A number of participants noted that the professional silos within the mental health sector constrain partnerships. Partnerships were noted as occurring, but "not in an integrated manner", with teams unaware of other teams' work.

\section{Chronosystem}

Participants' accounts of partnerships with consumer researchers interacted with the dimension of time in several ways; as has already been discussed in relation to time as a 
resource throughout this results section. Two more specific ways in which time was salient to participants' experiences of partnership related to immediate time demands, and more longterm career trajectories.

\section{Immediate time demands}

Many participants discussed ways collaborative teaching between consumer academics and other mental health academics facilitated positive relationships for research. However, the routine timelines for coursework and clinical placements sometimes added another barrier to research. For instance, one participant referred to an annual event where consumer researchers get together (which allowed for bringing together teams as in Extract 5). For one team, though, the event coincided with preparation of students for clinical placements, leading to several colleagues missing out.

\section{Career life courses}

Mental health research career processes at universities tend to be structured around mainstream notions of life-course pathways in education and work. Consumers commonly entered into research later in life due to their life experiences in relation to mental illness, as discussed in Extract 9. The relatively late entry in the research arena seemed to work against the efforts of researchers, both consumer and other, in developing consumer research in general.

\section{Extract 9}

"..a lot of people will have done some work to get to wanting to be somebody who is identified as a person using the service. They'll have whatever experiences they've had to bring them to that point, which means that their start in a research career might be later. And so, I think there's a barrier that people, they might need a decent income. They can't be a research student on a $\mathrm{PhD}$ scholarship. So, people's requirements for a decent earning might be, like a mid-career person, but actually in terms of research they're not mid-career. They're early career." 
Several participants referred to this issue, particularly in relation to the complexity of consumers needing to balance other paid work in addition to their research activities for a liveable income. Career progression was also seen as an issue for other mental health researchers. Some participants noted that collaborations with consumer researchers was not valued enough by senior academics when applying for promotion, for instance. These factors together inhibit the development of consumer research as a discipline.

$\square$

\section{DISCUSSION}

The findings of the current study highlight the structures that impact upon collaborative relationships between consumer researchers and other mental health researchers. Drawing on ecological systems theory (Darling, 2007), these structures include contexts of the microsystem (such as practical considerations necessary to build collaborative relationships), the meso- and exo-systems (such as negotiating timelines flexibly, and issues related to external deadlines set by funding bodies), the macrosystem (such as silos in health research), and the chronosystem (including career timelines and immediate time demands). These findings elaborate on some of the systemic obstacles across and within layers of the ecosystem frequently observed in collaborations with consumer researchers. There are also several opportunities that the interview data highlight: ways that more experienced researchers can ally with emerging researchers, how newer technology might overcome some of the microsystemic barriers to building relationships, and how greater flexibility might lead to better research outcomes and collaborations.

Previous literature has explored how relationship building and genuine trust is crucial to collaboration (Hoonakker, Pecanac, Brown, \& Carayon, 2017). The current study extends this work through the application of ecological systems theory. Collaboration is primarily experienced at the microsystemic level of the immediate relationship and research outputs, however there are several broader barriers and facilitators which also impact on the relationship.

Popular discourse within the field often assumes that one of the major barriers to collaborations with consumer researchers is that their mental ill health impedes their ability to meet deadlines (Gee et al., 2016; Ipes, Lucas, Phelan, \& White, 2016). The current findings challenge such dominant ideas, and suggest that systemic issues pose much more formidable barriers. Further, participants in the current study note that often consumers have much healthier attitudes towards systemic demands. In Extract 6, we present the words of one 
participant, who argues that it should be considered a strength that consumer researcher collaborators are better able to set boundaries around their work role than other collaborators. Our findings challenge existing opinions that assume that consumers' individual contexts are the major barriers to collaboration (Gee et al., 2016), and instead point to issues in the broader layers of the ecosystem which require attention.

The findings of this study have multiple implications to inform strengthened collaborations between consumer researchers and other mental health researchers. First, the inflexibility that causes difficulties in the microsystem of the collaborative relationship is related to some of the broader structural contexts, such as funding deadlines and other bureaucratic demands. Although it is often not within individual researchers' ability to control these exo- or macro-systemic factors, critical thought is needed in regards to how they can be challenged at the microsystemic level. For example, being able to plan research funding applications in adyance so that last-minute rushes are avoided, or being more flexible about working hours can be controlled to a certain degree, thereby inoculating against broader systemic pressures as much as possible.

Second, a chronosystemic barrier to greater collaboration with consumer researchers (and, as a result a barrier to a larger number of consumer researchers working in the field) is related to career life courses. As described by participants (such as in Extract 9), consumer researchers often start research careers 'later', and financial needs and obligations can constrain research training. Other mental health researchers might think critically about how to ensure consumer researchers access equitable opportunities for research training and track record development. Drawing on existing undergraduate programmes to retain minority group students in university study (Beech et al., 2013), programmes designed to encourage and upskill consumers could be implemented, to challenge the broader systemic barriers of research careers for consumers. Similarly, other mental health researchers with more developed track records might do what they can to create positions for consumer researchers in their departments, or to build consumer researchers into research projects in early stages including in funding opportunities.

Third, relationship building between consumer researchers and other mental health researchers is essential for enhanced participation, and fostering more consumer-led and coproduced research. Some of the participants elaborated on ways of 'bringing together' collaborators. Previous research has considered the way that informal relationship building 
such as meeting over coffee or dancing at conferences increases relational congruence (Lu \& Scholz, 2016). The current study extends this finding by suggesting that collaborators can find innovative ways of 'bringing together' within research microsystems, such as constructing virtual groups (as in Extract 5) to facilitate partnerships despite distances between people. It is likely that this would also benefit other researchers who have wanted to engage in better co-produced research but who are remote or otherwise not connected to consumer researchers. Developing a virtual network of consumer researchers could also assist other researchers to form new partnerships with consumer researchers, each with relevant expertise in specific domains of mental health systems.

A potential limitation of this current study is that participants who agreed to participate are other mental health researchers more likely to be receptive to collaboration with consumer researchers. Our analytic lens has centred on the relationship between consumer researchers and other mental health researchers, but the majority of other mental health researchers more broadly have not collaborated with consumer researchers, and do not see consumer researchers as central to mental health research. Therefore, it seems likely that other ecosystemic barriers to meaningful relationships would include attitudinal and ideological resistance to consumer researchers and consumer research.

\section{CONCLUSION}

The ecosystem surrounding collaborations between consumer researchers and other mental health researchers comprises structures that impact upon the collaborative relationship. The current study provides an overview of various structures at each level of the ecosystem. Future research might consider how mental health researchers can start to challenge broader systemic barriers so that the collaborative relationship, or the microsystem, facilitates better inclusion of and partnership with consumers in the research process.

\section{RELEVANCE FOR CLINICAL PRACTICE}

Best clinical practice is based on research that is relevant to those who experience mental ill health and mental health service usage. Research that has been conducted with active and meaningful involvement of consumers, is therefore most likely to ensure clinical practice is safe, meets consumers' needs, and improves the quality of care provided.

\section{References}


Beech, B. M., Calles-Escandon, J., Hairston, K. G., Langdon, M. S. E., Latham-Sadler, B. A., \& Bell, R. A. (2013). Mentoring programs for underrepresented minority faculty in academic medical centers: a systematic review of the literature. Academic medicine: journal of the Association of American Medical Colleges, 88(4). doi:10.1097/ACM.0b013e31828589e3

Braun, V., \& Clarke, V. (2006). Using thematic analysis in psychology. Qualitative Research in Psychology, 3, 77-101.

Bryant, W., Parsonage, J., Tibbs, A., Andrews, C., Clark, J., \& Franco, L. (2012). Meeting in the mist: key considerations in a collaborative research partnership with people with mental health issues. Work, 43(1), 23-31. doi:10.3233/WOR-2012-1444

Case, A. D., Byrd, R., Claggett, E., DeVeaux, S., Perkins, R., Huang, C., . . Kaufman, J. S. (2014). Stakeholders' perspectives on community-based participatory research to enhance mental health services. American Journal of Community Psychology, 54(34), 397-408. doi:10.1007/s10464-014-9677-8

Darling, N. (2007). Ecological systems theory: The person in the center of the circles. Research in human development, 4(3-4), 203-217. doi:10.1080/15427600701663023

Fothergill, A., Mitchell, B., Lipp, A., \& Nortway, R. (2012). Setting up a mental health user research group: a process paper. Journal of Research in Nursing, 18(8), 746-759. doi:10.1177/1744987112451579

Gee, A., McGarty, C., \& Banfield, M. (2016). Barriers to genuine consumer and carer participation from the perspectives of Australian systemic mental health advocates. Journal of Mental Health, 25(3), 231-237. doi:10.3109/09638237.2015.1124383

Graham, T., Rose, D., Murray, J., Ashworth, M., \& Tylee, A. (2014). User-generated quality standards for youth mental health in primary care: a participatory research design using mixed methods. British Medical Journal: Quality and Safety, 23(10), 857-866. doi:10.1136/bmjqs-2014-002842

Happell, B., \& Scholz, B. (2018). Doing what we can but knowing our place: Being an ally to promote consumer leadership in mental health. International Journal of Mental Health, 27(1), 440-447. doi:10.1111/inm.12404 
Hoonakker, P. L., Pecanac, K. E., Brown, R. L., \& Carayon, P. (2017). Virtual collaboration, satisfaction, and trust between nurses in the tele-ICU and ICUs: Results of a multilevel analysis. Journal of critical care, 37(February), 224-229. doi:10.1016/j.jcrc.2016.10.018

INVOLVE. (2012). Briefing notes for researchers: public involvement in NHS, public health and social care research. Eastleigh: Involve.

Ipes, C., Lucas, J., Phelan, J. C., \& White, R. C. (2016). The stigma of mental illness in the labor market. Social Science Research, 58, 16-25. doi:10.1016/j.ssresearch.2015.12.001

Juntanamalaga, P., Roper, C., Happell, B., \& Scholz, B. (2019). “They can’t empower us”: The role of allies in the consumer movement. International Journal of Mental Health Nursing.

Landry, D. (2017). Survivor research in Canada: 'talking' recovery, resisting psychiatry, and reclaiming madness. Disability and Society, 32(9), 1437-1457. doi:10.1080/09687599.2017.1322499

Lowes, L., \& Hulatt, I. (2005). Involving service users in health and social care research. Abingdon, OX: Taylor and Francis.

Lu, V. N., \& Scholz, B. (2016). Knowing me, knowing you: Mentorship, friendship, and dancing queens. In C. Plewa \& J. Conduit (Eds.), Making a difference through marketing: A quest for diverse perspectives (pp. 201-210). Singopare: Springer. doi:10.1007/978-981-10-0464-3_15

Michalak, E. E., Jones, S., Lobban, F., Algorta, G. P., Barnes, S. J., Berk, L., . . CREST.BD. (2016). Harnessing the potential of community-based participatory research approaches in bipolar disorder. International Journal of Bipolar Disorders20164, 4(4), 1-9. doi:10.1186/s40345-016-0045-5

Minogue, V., \& Girdlestone, J. (2010). Building capacity for service user and carer involvement in research: the implications and impact of best research for best health. International Journal of Health Care Quality Assurance, 23(4), 422-435. doi:10.1108/09526861011037470 
Mjosund, N. H., Eriksson, M., Espnes, G. A., Haaland-Overby, M., Jensen, S. L., Norheim, I., . . Vinje, H. F. (2017). Service user involvement enhanced the research quality in a study using interpretative phenomenological analysis - the power of multiple perspectives. Journal of Advanced Nursing, 73(1), 265-278. doi:10.1111/jan.13093

Ochocka, J., Janzen, R., \& Nelson, G. (2002). Sharing power and knowledge: professional and mental health consumer/survivor researchers working together in a participatory action research project. . Psychiatric Rehabilitation Journal, 25(4), 379-387.

Patterson, S., Trite, J., \& Weaver, T. (2014). Activity and views of service users involved in mental health research: UK survey. British Journal of Psychiatry, 205(1), 68-75. doi:10.1192/bjp.bp.113.128637

Potter, J. (2012). Discourse analysis and discursive psychology. In H. Cooper (Ed.), APA Handbook of Research Methods in Psychology: Vol. 2. Quantitative, Qualitative, Neuropsychological, and Biological (pp. 111-130). Washington: APA Press.

Roper, C., Grey, F., \& Cadogan, E. (2018). Co-production - Putting principles into practice in mental health contexts. Retrieved from http://recoverylibrary.unimelb.edu.au/_data/assets/pdf_file/0010/2659969/Coproduct ion putting-principles-into-practice.pdf

Rose, D. (2017). Service user / survivor-led research in mental health: Epistemological possibilities. Disability \& Society, 32(6), 773-789. doi:10.1080/09687599.2017.1320270

Schneider, B. (2012). Participatory action research, mental health service user research, and the hearing (our) voices projects. International Journal of Qualitative Methods, 11, $152-165$.

Scholz, B., Bocking, J., \& Happell, B. (2017a). Breaking through the glass ceiling: Consumers in mental health organisations' hierarchies. Issues in Mental Health Nursing, 38(5), 374-380. doi:10.1080/01612840.2017.1280106

Scholz, B., Bocking, J., \& Happell, B. (2017b). Improving exchange with consumers within mental health organizations: Recognizing mental ill health experience as a 'sneaky, 
special degree'. International Journal of Mental Health Nursing. doi:10.1111/inm.12312

Scholz, B., Stewart, S. J., Bocking, J., \& Happell, B. (2017). Rhetoric of representation: The disempowerment and empowerment of consumer leaders. Health Promotion International, 34(1), 166-174. doi:10.1093/heapro/dax070

Staley, K. (2012). An evaluation of service user involvement in studies adopted by the Mental Health Research Network. London: Mental Health Research Network.

Stebbins, R. (2001). Exploratory research in the social sciences: Qualitative research methods: Volume 48. London, England: Sage.

Veseth, M., Binder, P. E., Borg, M., \& Davidson, L. (2017). Collaborating to stay open and aware: seryice user involvement in mental health research as an aid in reflexivity. Nordic Psychology, 69(4), 256-263. doi:10.1080/19012276.2017.1282324

Wallcraft, J., Schrank, B., \& Amering, M. (2009). Handbook of service user involvement in mental health research. Chichester: Wiley.

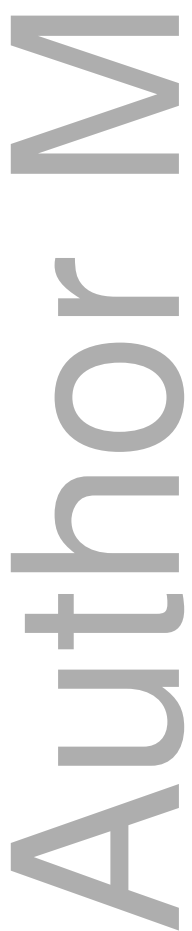




\section{University Library}

\section{- M M N E R VA A gateway to Melbourne's research publications}

Minerva Access is the Institutional Repository of The University of Melbourne

Author/s:

Scholz, B;Gordon, S;Bocking, J;Liggins, J;Ellis, P;Roper, C;Platania-Phung, C;Happell, B

Title:

'There's just no flexibility': How space and time impact mental health consumer research

Date:

2019-08-01

Citation:

Scholz, B., Gordon, S., Bocking, J., Liggins, J., Ellis, P., Roper, C., Platania-Phung, C. \& Happell, B. (2019). 'There's just no flexibility': How space and time impact mental health consumer research. INTERNATIONAL JOURNAL OF MENTAL HEALTH NURSING, 28 (4), pp.899-908. https://doi.org/10.1111/inm.12589.

Persistent Link:

http://hdl.handle.net/11343/285645 